\title{
Cardiac Magnetic Resonance Imaging - A Predictor Tool for Sudden Cardiac Death
}

\author{
NILUFAR FATEMA ${ }^{1}$, MD. ABU SIDDIQUE ${ }^{1}$, SAJAL KRISHNABANERJEE ${ }^{1}$, SYDE ALI AHSAN ${ }^{1}$, FAZLUR RAHMAN ${ }^{1}$, \\ CHAUDHURY MESHKAT AHMED ${ }^{1}$, M AMUQEET ${ }^{1}$, MOHAMMAD ATIQUR RAHMAN ${ }^{2}$, KHURSHED AHMED $^{1}$, \\ ELORA SHARMIN ${ }^{1}$, SHEIKH FOYEZ AHMED ${ }^{1}$, MD. ASHRAF UDDIN SULTAN ${ }^{1}$, SHEIKH MUHAMMAD ABU BAKAR ${ }^{3}$ \\ ${ }^{1}$ Department of Cardiology, Bangabandhu Sheikh Mujib Medical University, Shahbah, Dhaka, ${ }^{2}$ Department of Medicine, Bangabandhu \\ Sheikh Mujib Medical University, Shahbah, Dhaka, ${ }^{3}$ Department of Anatomy, Ibrahim Medical College, Shahbag, Dhaka
}

Address for correspondence: Dr. Nilufar Fatema, Consultant, Department of Cardiology, Bangabandhu Sheikh Mujib Medical University, Shahbah, Dhaka. E-mail: nilufar.fatema@gmail.com

\begin{abstract}
:
Cardiovascular magnetic resonance (CMR) detects myocardial fibrosis, which appears as late gadolinium enhancement (LGE) after gadolinium contrast administration and may convey prognostic importance. Myocardial fibrosis is the source of malignant arrhythmia like ventricular tachycardia or ventricular fibrillation. Sudden cardiac death occurs in these group patients. By detecting myocardial fibrosis we can select these groups of patients for implantable cardioverter defibrillator. Thus sudden cardiac death can be prevented in some extent.
\end{abstract}

Keywords: Cardiac MRI, Sudden cardiac death.

\section{Introduction:}

Patients with nonischemic cardiomyopathy (NICM) comprise one-third of the heart failure (HF) population and are at risk for significant morbidity and mortality. ${ }^{1,2}$ NICM is the most common indication for heart transplantation. Ten-year survival is below 60\% with deaths often preceded by frequent hospitalizations for HF exacerbations. Forty percent of deaths are sudden cardiac death (SCD) and placement of an ICD for primary prevention reduces arrhythmic deaths in NICM patients with left ventricular ejection fraction (LVEF) $\leq 35 \%$. $^{3}$ However, risk stratification remains challenging, particularly in the individual patient in whom the clinical course frequently correlates poorly with LVEF. Better riskstratification tools might allow earlier intervention in highrisk patients, improving both quality of life and survival. In patients with NICM, myocardial fibrosis has been identified pathologically. Macroscopic regions of fibrosis have also been visualized by cardiovascular magnetic resonance (CMR), appearing as areas of late gadolinium enhancement (LGE). Increasing amounts of fibrosis potentially result in increased LV stiffness and reduced LV compliance, thereby progressively impairing both diastolic and systolic function, reducing cardiac output. One trail showed that the presence and extent of fibrosis in CMR LGE are associated with a higher risk of adverse cardiac outcomes in patients with NICM and LVEF $\leq 35 \%$.

\section{Sudden cardiac death (SCD):}

SCD is the most common single cause of death in world. Yearly, worldwide, more than three million people will die due to SCD, the majority because of ventricular arrhythmia (VA). ${ }^{4}$ It is possible to protect patients who are at risk for life-threatening VA and subsequent SCD with the implantation of the implantable cardioverter defibrillator (ICD). 5,6 A low left ventricular ejection fraction (LVEF) is considered to be the most important risk factor in these cases. LVEF measured at rest has an important impact on long-term prognosis. The frequency of SCD increases with a decrease in LVEF. ${ }^{7}$ ICD implantation improved survival in patients with a prior myocardial infarction and an LVEF $<30 \%$ in compared to conventional pharmacological treatment (amiodarone). ${ }^{6}$ ICD showed superior result in patients group with all-cause cardiomyopathy in combination with mild to moderate heart failure (NYHA class II or III) and an LVEF $<35 \%$ than antiarrhythmic group. ${ }^{8}$ These results form the basis of the current ACC/ $\mathrm{AHH} / \mathrm{ESC}$ guidelines for the management of patients with VA and prevention of SCD. In these guidelines, the use of echocardiography is advised to measure LVEF. ${ }^{9}$ Twodimensional echocardiography is the most widely used method to assess LVEF. This technique allows real-time imaging of the function and anatomy of the heart, is readily available, inexpensive and non-invasive. Important limitations are its operator dependency, acoustic window dependency, moderate reproducibility and accuracy. 8,9 When accurate assessment by echocardiography is not feasible, other imaging modalities can be used. ${ }^{9}$

Cardiac magnetic resonance imaging (CMR):

CMR is one of the most accurate imaging methods. ${ }^{10,11}$ LVEF is measured by echocardiography, CMR, 

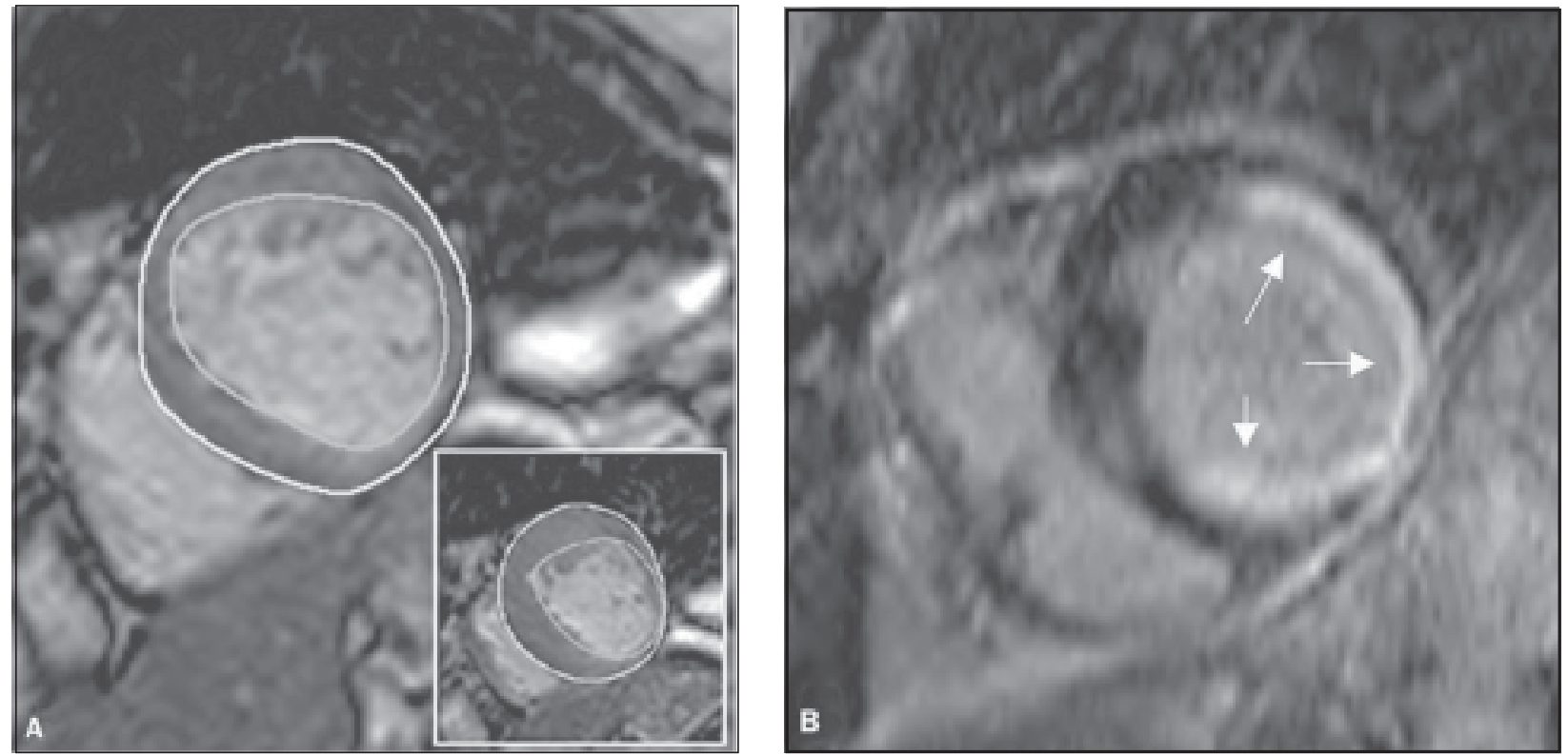

Fig.-1: Magnetic resonance image. A) Measurement of LVEF in a patient with prior myocardial infarction. Short-axis slices enddiastolic and end-systolic (frame). B) Example of contrast( Gadolinium)-enhanced MRI. Short-axis view shows a large hyperenhanced area (arrows).

radionuclide ventriculography (RV) or computed tomography (CT). In echocardiography, measurement of LVEF varies operator to operator. Sometime cardiologists use geometric assumption while doing echo which is variable. So CMR is very useful in Cardiomyopathy. ${ }^{12,13}$ The reproducibility and accuracy of LVEF calculation by CMR are higher than echocardiography both in normal and dilated heart. ${ }^{12,14,15}$ Calculation of LVEF by RV has been used for more than two decades. Advantages of RV include high reproducibility and low inter and intraobserver variability. ${ }^{16,17}$ In $95 \%$ of the cases, results of repeated quantitative radionuclide assessment of LVEF can be expected to be within a $2 \%$ and $4 \%$ range. By using the CT, calculation LVEF takes about ten minutes which is faster. This technique is useful in both normal and abnormal hearts. Its reproducibility and accuracy is comparable with the accuracy of CMR. But CT is limited by radiation exposure and the use of iodated contrast material with additional risks. ${ }^{18} \mathrm{CMR}$ can detect particular area of fibrosis whether it is regional or mid layer of myocardium in late gadolinium enhancement stage. Regional fibrosis indicates myocardial infarction and global mid layer of fibrosis suggests cardiomyopathy.

\section{Cardiac MRI protocol:}

Prior to ICD implantation, patients may underwent CMR. Cine images were acquired with a steady-state free precession pulse sequence (TR 2.5-3.8, TE 1.1-1.6, average in-plane resolution $1.5 \times 2.4 \mathrm{~mm}$, flip angle $45-60^{\circ}$, temporal resolution 40-45 msec) in long-axis planes and contiguous 8-mm short-axis slices from the mitral annulus to the apex. Fifteen to thirty minutes after intravenous administration of $0.2 \mathrm{mmol} / \mathrm{kg}$ gadodiamide, delayed contrast enhanced images can be acquired using inversion-recovery fast gradient-echo pulse sequences in the same short-axis locations as the cine images. In order to exclude artifact, short axis imaging was repeated in 2 different phaseencoding directions and cross-sectional long axis views were also acquired. Imaging parameters were: TR 5.4-8.3 ms, TE 1.3-3.9 ms, average in-plane spatial resolution 1.41.5×2.2-2.4 mm, 8-mm slice thickness, 2-mm gap, TI 175$300 \mathrm{msec}$ (adjusted as needed in the delayed enhancement image acquisitions to optimally null the signal of normal myocardium), 1-2 R-R imaging, flip angle 20-25․ ${ }^{19}$

\section{Conclusion:}

The presence of CMR LGE identifies a group of NICM patients with an eightfold higher risk of an index composite outcome of heart failure hospitalization, appropriate action of ICD and cardiac death, compared to patients without LGE. CMR scanning and interpretation are relatively straightforward and can be performed at many hospitals which routinely provide care for patients with heart disease. Future more research will be needed to determine CMR findings can lower morbidity and mortality for patients with nonischemic cardiomyopathy by considering ICD or CRT or left ventricular assist device placement. As these devices are expensive it would be cost effective if correct selection of patients. 


\section{References:}

1. Hunt SA. ACC/AHA 2005 guideline update for the diagnosis and management of chronic heart failure in the adult: a report of the American College of Cardiology/American Heart Association Task Force on Practice Guidelines (Writing Committee to Update the 2001 Guidelines for the Evaluation and Management of Heart Failure). J Am Coll Cardiol 2005;46:e1-82.

2. Maron BJ, Towbin JA, Thiene G, et al. Contemporary definitions and classification of the cardiomyopathies: an American Heart Association Scientific Statement from the Council on Clinical Cardiology, Heart Failure and Transplantation Committee; Quality of Care and Outcomes Research and Functional Genomics and Translational Biology Interdisciplinary Working Groups; and Council on Epidemiology and Prevention. Circulation 2006;113:180716.

3. Kadish A, Dyer A, Daubert JP, et al. Prophylactic defibrillator implantation in patients with nonischemic dilated cardiomyopathy. N Engl J Med 2004;350:2151-58.

4. Van Royen N, Jaffe CC, Krumholz HM, Johnson KM, Lynch PJ, Natale D, et al. Comparison and reproducibility of visual echocardiographic and quantitative radionuclide left ventricular ejection fractions. Am J Cardiol 1996;77:843-50.

5. Van der Wall EE, Bax JJ. Different imaging approaches in the assessment of left ventricular dysfunction: all things equal? Eur Heart J 2000;21:1295-97.

6. Belge B, Coche E, Pasquet A, Vanoverschelde JL, Gerber BL. Accurate estimation of global and regional cardiac function by retrospectively gated multidetector row computed tomography: comparison with cine magnetic resonance imaging. Eur Radiol 2006;16:1424-33.

7. Josephson M, Wellens HJ. Implantable defibrillators and sudden cardiac death. Circulation 2004;109:2685-91.

8. A comparison of antiarrhythmic-drug therapy with implantable defibrillators in patients resuscitated from nearfatal ventricular arrhythmias. The Antiarrhythmics versus Implantable Defibrillators (AVID) Investigators. $N$ Engl $J$ Med 1997;337:1576-83.

9. Moss AJ, Zareba W, Hall WJ, Klein H, Wilber DJ, Cannom DS et al. Prophylactic implantation of a defibrillator in patients with myocardial infarction and reduced ejection fraction. $N$ Engl J Med 2002;346:877-83.
10. Grothues F, Smith GC, Moon JC, Bellenger NG, Collins P, Klein HU, et al. Comparison of interstudy reproducibility of cardiovascular magnetic resonance with two-dimensional echocardiography in normal subjects and in patients with heart failure or left ventricular hypertrophy. Am J Cardiol 2002;90: 29-34.

11. Alfakih K, Reid S, Jones T, Sivananthan M. Assessment of ventricular function and mass by cardiac magnetic resonance imaging. Eur Radiol 2004;14:1813-22.

12. Bellenger NG, Burgess MI, Ray SG, Lahiri A, Coats AJ, Cleland JG, et al. Comparison of left ventricular ejection fraction and volumes in heart failure by echocardiography, radionuclide ventriculography and cardiovascular magnetic resonance; are they interchangeable? Eur Heart J 2000;21:1387-96.

13. Walsh TF, Hundley WG. Assessment of ventricular function with cardiovascular magnetic resonance. Cardiol Clin 2007;25: 15-33.

14. Hoffmann R, von Bardeleben S, ten Cate F, Borges AC, Kasprzak J, Firschke C, et al. Assessment of systolic left ventricular function: a multi-centre comparison of cineventriculography, cardiac magnetic resonance imaging, unenhanced and contrast-enhanced echocardiography. Eur Heart J 2005;26:607-16.

15. G rothues F, Smith GC, Moon JC, Bellenger NG, Collins P, Klein HU, et al. Comparison of interstudy reproducibility of cardiovascular magnetic resonance with two-dimensional echocardiography in normal subjects and in patients with heart failure or left ventricular hypertrophy. Am J Cardiol 2002;90:29-34.

16. Van Royen N, Jaffe CC, Krumholz HM, Johnson KM, Lynch PJ, Natale D, et al. Comparison and reproducibility of visual echocardiographic and quantitative radionuclide left ventricular ejection fractions. Am J Cardiol 1996;77:843-50.

17. Van der Wall EE, Bax JJ. Different imaging approaches in the assessment of left ventricular dysfunction: all things equal? Eur Heart J 2000;21:1295-97.

18. Belge B, Coche E, Pasquet A, Vanoverschelde JL, Gerber BL. Accurate estimation of global and regional cardiac function by retrospectively gated multidetector row computed tomography: comparison with cine magnetic resonance imaging. Eur Radiol 2006;16:1424-33.

19. Simonetti OP, Kim RJ, Fieno DS, et al. An improved MR imaging technique for the visualization of myocardial infarction. Radiology 2001;218:215-23. 